\title{
Chordal Topologies for Interconnection Networks *
}

\author{
Ramón Beivide ${ }^{1}$, Carmen Martínez ${ }^{1}$, Cruz Izu ${ }^{2}$, Jaime Gutierrez ${ }^{1}$, \\ José-Ángel Gregorio ${ }^{1}$ and José Miguel-Alonso ${ }^{3}$ \\ 1 Universidad de Cantabria. Avenida de los Castros s/n. 39005 Santander, Spain. \\ mon@atc.unican.es \\ 2 The University of Adelaide. 5005 Adelaide, South Australia. \\ 3 Univ. del País Vasco UPV/EHU. P. M. Lardizabal, 1, 20018 San Sebastián, Spain.
}

\begin{abstract}
The class of dense circulant graphs of degree four with optimal distance-related properties is analyzed in this paper. An algebraic study of this class is done. Two geometric characterizations are given, one in the plane and other in the space. Both characterizations facilitate the analysis of their topological properties and corroborate their suitability for implementing interconnection networks for distributed and parallel computers. Also a distance-hereditary non-disjoint decomposition of these graphs into rings is computed. Besides its practical consequences, this decomposition allows us the presentation of these optimal circulant graphs as a particular evolution of the traditional ring topology.
\end{abstract}

\section{Introduction}

Ring topologies have frequently been used to implement local and campus area networks as well as other interconnection subsystems for diverse digital devices. In order to improve their relatively poor performance and survivability, extensions of rings such as Chordal Rings of degree three and four have been considered in the literature [1],[5]. The Torus is another low-degree regular network, based on a collection of rings, often used to interconnect highly coupled parallel systems. Some of the latest parallel computers using this topology are the IBM BlueGene/L supercomputer [2] and different multiprocessor servers based on the Alpha 21364 microprocessor [13].

Interconnection networks can be modelled by graphs with vertices representing their processing elements and edges representing the communication links between them. The performance and robustness of any network are mostly determined by the topological characteristics of its underlying graph.

Circulant graphs have deserved significant attention during the last decades, the traditional ring and the complete graph topologies belong to this class of graphs. Circulants of different degrees constituted the basis of some classical

\footnotetext{
* This work has been partially supported by the Spanish CICYT project TIC20010591-C02-01 and by the Spanish Ministry of Education under grants FP-20012482,PR2002-0043 and BFM2001-1294.
} 
distributed and parallel systems [15], [7]. The design of certain data alignment networks for complex memory systems have also relied on circulant graphs [16].

A class of circulant graphs of degree four with minimal topological distances, denoted as Midimew networks, was presented in [4] as a basis for building optimal interconnection networks for parallel computers. One Midimew network exists for any given number of nodes, $N$, which is completely defined by means of a single parameter, as described later. These graphs are optimal because they have the minimum average distance among all circulant graphs of degree four; consequently, the diameter of a Midimew graph is also minimum. In addition, these graphs are regular, vertex-symmetric and maximally connected, which means that they possess a very high degree of fault tolerance. Optimal VLSI layouts for these networks have also been explored [9] and some instances of Midimew graphs have recently been used as a basis for designing networks for massively parallel computers [17]. It is known that some instances of Midimew graphs are isomorphic to Chordal Rings of degree four, thus sharing their topological distance properties; however, there exist infinite values of $N$ for which Midimew networks have smaller diameter and smaller average distance [5].

In this paper, we focus exclusively on dense circulant graphs of degree four. For every integer $k$, there exists a dense optimal circulant graph composed of $2 k^{2}+2 k+1$ nodes, being $k$ the diameter of the graph. In section 2 we prove that any optimal dense circulant graph of degree four with diameter $k$ is isomorphic to a Chordal Ring whose chord has length $2 k+1$. In particular, the corresponding Midimew graph is also isomorphic to this Chordal Ring. Section 3 is devoted to show two geometric characterizations of these optimal graphs that facilitate the analysis of their properties, especially those related to topological distances. As a by-product of the above-mentioned geometric characterizations, we present in the last section a distance-hereditary decomposition of dense optimal Chordal Rings into a set of traditional rings. All the rings belonging to this set have the same number of nodes and their diameter corresponds to the diameter of the Chordal Ring in which they are embedded. Moreover they preserve the minimal routing of the original circulant graph.

\section{Dense Optimal Chordal Rings of Degree Four}

A circulant graph with $N$ vertices and jumps $\left\{j_{1}, j_{2}, \ldots j_{m}\right\}$ is an undirected graph in which each vertex $n, 0 \leq n \leq N$, is adjacent to all the vertices $n \pm j_{i}$, with $1 \leq i \leq m$. We denote this graph as $C_{N}\left(j_{1}, j_{2}, \ldots j_{m}\right)$. The family of circulant graphs includes the complete graph and the cyclic graph (ring) among its members.

We say that a circulant is dense if it has the maximum possible number of nodes for a given diameter $k$. Thus, if $k$ is a positive integer, a dense ring or $C_{N}(1)$ of degree two has $N=2 k+1$ nodes. There is another non-dense ring with $2 k$ nodes. When adding another jump to the list, every integer $k$ defines a family of $4 k$ optimal (with minimum average distance and therefore minimum diameter) $C_{N}\left(j_{1}, j_{2}\right)$ graphs or Midimew networks of diameter $k$, with $j_{1}=b-1$ and $j_{2}=b$, 
where $b=\sqrt{\frac{N}{2}}$, [4]. The dense member of this family contains $2 k^{2}+2 k+1$ nodes. The other $4 k-1$ values of $N$ in the family correspond to non-dense graphs.

Two graphs $G$ and $H$ are isomorphic $(G \cong H)$ if there exists a bijective mapping between their node sets which preserves adjacency. Is easy to prove the following:

Lemma 1. Let $k$ be a positive integer, $N=2 k^{2}+2 k+1$. If $C_{N}(c, d)$ is an optimal circulant graph, then $C_{N}(c, d) \cong C_{N}(k, k+1)$.

Now, we consider the Chordal Ring $C_{N}(1,2 k+1)$. This Chordal Ring is isomorphic to the dense Midimew graph and hence, it is also optimal. To prove this claim we are going to use the following well-known theorem [1]:

Theorem 1. Let $N$ be a natural number. We have $C_{N}\left(j_{1}, j_{2}\right) \cong C_{N}\left(i_{1}, i_{2}\right)$ if and only if there exists an integer $u$ such that $\operatorname{gcd}(u, N)=1$ and $u\left\{ \pm j_{1}, \pm j_{2}\right\}=$ $\left\{ \pm i_{1}, \pm i_{2}\right\} \bmod N$.

Hence, the element $u=(k+1)^{-1}=-2 k$ provides the adequate isomorphism between the two graphs.

For the rest of the paper we are going to consider $C_{N}(1,2 k+1)$ Chordal Rings as the representative members of the class of dense optimal circulant graphs of degree four.

\section{Geometrical Characterization of Optimal Chordal Rings}

In this section we will present two geometrical characterizations of $C_{N}(1,2 k+1)$ optimal graphs, in two and three dimensions, that lead to two physical implementations of interconnection networks based on these graphs.

Let us begin with the bi-dimensional geometric characterization. The vertexsymmetry of graphs allows their analysis from any vertex; node 0 will be used in the rest of the paper. In these graphs, there are $4 d$ different nodes at distance $d$ from node 0 , with $1 \leq d \leq k$, and consequently, the total number of nodes of the graph is $N=k^{2}+(k+1)^{2}$. It is known that certain families of graphs can be fully represented by plane tessellations when their nodes are associated with regular polygons [7]. If each node in a $C_{N}(1,2 k+1)$ graph is associated with a unitary square with its four neighbours attached to its sides as in [15], the graph can be characterized by a serrated or discrete square tile of area $N$, like the one in Fig. 1. This tile tessellates the plane, and the network wrap-around links are defined according to the periodical pattern dictated by such a tessellation.

A popular geometric proof of the Pythagorean theorem, which is clearly reflected in the equation $N=k^{2}+(k+1)^{2}$, shows the square of the hypotenuse of a right-angled triangle of legs $k$ and $k+1$ as composed of four copies of such a right-angled triangle plus a central square with side $k+1-k=1$. The tile in Fig. 1 can be seen as a geometrical proof of a discrete version of the Pythagorean Theorem. In this discrete version, the right-angled triangles with legs $k$ and $k+1$ 


\begin{tabular}{|l|l|l|l|l|l|l|l|l|l|l|}
\hline 20 & 2 & 9 & 16 & 23 & 5 & 12 & 19 & 1 & 8 & 15 \\
\hline 19 & 1 & 8 & 15 & 22 & 4 & 11 & 18 & 0 & 7 & 14 \\
\hline 18 & 0 & 7 & 14 & 2 & 3 & 10 & 17 & 24 & 6 & 13 \\
\hline 17 & 24 & 6 & 13 & 20 & 2 & 9 & 16 & 23 & 5 & 12 \\
\hline 16 & 23 & 5 & 12 & 19 & 1 & 8 & 15 & 22 & 4 & 11 \\
\hline 15 & 22 & 0 & 11 & 18 & 0 & 7 & 14 & 21 & 3 & 10 \\
\hline 14 & 21 & 3 & 10 & 17 & 24 & 6 & 13 & 20 & 2 & 9 \\
\hline 13 & 20 & 2 & 9 & 16 & 23 & 5 & 12 & 19 & 1 & 8 \\
\hline 12 & 19 & 1 & 8 & 15 & 22 & 4 & 11 & 18 & 0 & 7 \\
\hline 11 & 18 & 0 & 7 & 14 & 21 & 3 & 10 & 17 & 24 & 6 \\
\hline 10 & 17 & 24 & 6 & 13 & 20 & 2 & 9 & 16 & 23 & 5 \\
\hline
\end{tabular}

Fig. 1. Pythagorean Interpretation of a $C_{25}(1,7)$ Graph.

have been replaced by discrete right-angled triangles with both legs of length $k$, in which the hypotenuse adopts a serrated shape. The area of one of these rightangled triangles coincides with the area of its discrete version. Then, every dense optimal $C_{N}(1,2 k+1)$ graph is characterized by four copies of a discrete rightangled triangle with both legs of length $k$, plus a central unitary square. Such geometric interpretation, in terms of right-angled triangles, simplifies the study of the distance-related properties of these graphs.

Related also to another geometrical proof of the Pythagorean Theorem, a new layout for $C_{N}(1,2 k+1)$ graphs can be obtained as shown in Fig. 2.

This representation can be especially suitable for implementing interconnection networks for highly coupled parallel systems because it minimizes the number of wrap-around links. The graph is now seen as two attached squares with sides $k$ and $k+1$. The number of wrap-around links is equal to the semi-perimeter of the resulting polygon, which is $3 k+2$.

The rest of this Section introduces a three-dimensional graph transformation that naturally avoids the unbalance between internal mesh-like links and external wrap-around links. When technological advances permit it, our threedimensional layout should be one of the best choices for implementing an interconnection network based on circulant graphs with both minimal topological and physical distances among nodes.

The nodes of an optimal graph can be placed on the surface of a Torus with radii $R$ and $r$ by using its parametric equations, defined as follows: 


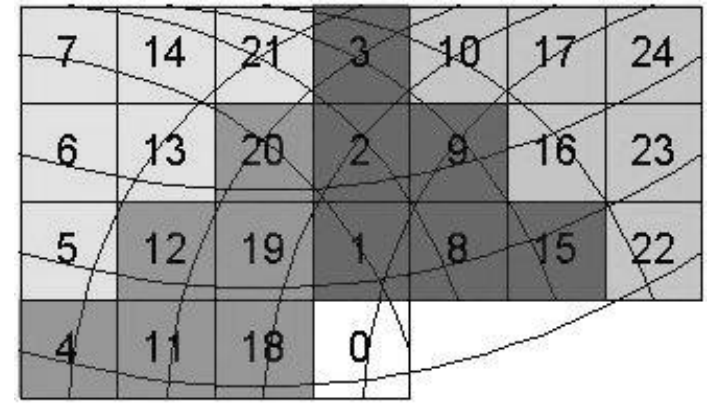

Fig. 2. Mesh-connected $C_{25}(1,7)$ Graph with Minimum Number of Wrap-around Links.

$$
\left\{\begin{array}{c}
x(u, v)=\cos (v)(R-r \cos (u)) \\
y(u, v)=\sin (v)(R-r \cos (u)) \quad u, v \in[0,2 \pi] \\
z(u, v)=r \sin (u)
\end{array}\right.
$$

If we define, as in [9], $u_{j}=\frac{2 \pi}{N} j$ and $v_{j}=\frac{2 \pi(2 k+1)}{N} j$, then every node $j$ of the graph, with $j \in\{0,1, \ldots, N-1\}$, corresponds to a point $T_{j}$ of the Torus surface by using the mapping $T_{j}=\Psi(j)=\left(x\left(u_{j}, v_{j}\right), y\left(u_{j}, v_{j}\right), z\left(u_{j}, v_{j}\right)\right)$. The points $T_{j}=\left(t_{x(j)}, t_{y(j)}, t_{z(j)}\right)$, being $t_{x}, t_{y}, t_{z}$ their Cartesian coordinates, are located on the helix $T(s ; 1,2 k+1)=\left(x\left(u_{s}, v_{s}\right), y\left(u_{s}, v_{s}\right), z\left(u_{s}, v_{s}\right)\right)$, where $s \in$ $[0, N-1]$. Similarly, the same points $T_{j}=\left(t_{x(j)}, t_{y(j)}, t_{z(j)}\right)$, are located on the curve $T(s ; 2 k+1,-1), s \in[0, N-1]$. The intersection points of $T(s ; 1,2 k+1)$ and $T(s ; 2 k+1,-1)$ are the points $T_{j}$, in which we locate every node $j$ of the graph. Actually, both curves define two disjoint Hamiltonian paths, which are always embedded in this class of graphs. When $R=2 r$, as shown in Fig. 3 for $C_{N}(1,2 k+1)$ with $k=3$, the length of all the graph edges is the same, being this length the closest value to the Euclidean distance between two neighbour nodes [9].

This embedding of the $C_{N}(1,2 k+1)$ graph on a Torus surface can lead to a $3 \mathrm{D}$ network implementation with all the physical links having the same minimum length and never crossing among them. In addition, the underlying graph has the minimal topological distances among all the circulants of degree four. Some designs for futuristic applications as the ones introduced in [10], suggest the use in the few next years of cylindrical structures and free-space optical communications for implementing extreme interconnection networks.

\section{Optimal Dense Chordal Rings as a Set of Rings}

It can be shown that dense optimal $C_{N}(1,2 k+1)$ graphs can be decomposed into a non-disjoint collection of $2 N$ rings that preserves the distance-related properties of the original graph. These rings, or circulants of degree two, are also dense and their diameter is the diameter of the circulant in which they are embedded. 


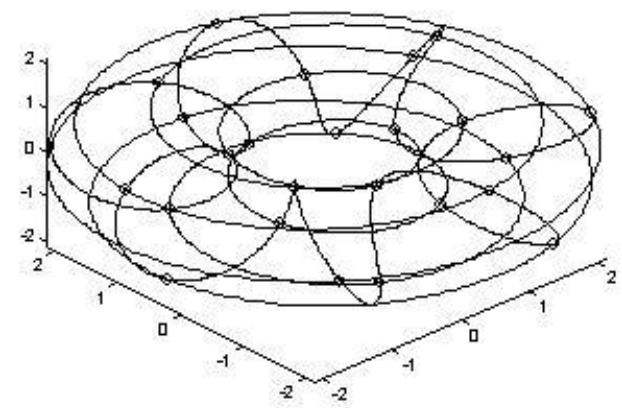

Fig. 3. 3D Torus Embedding of the $C_{25}(1,7)$ Graph.

In order to obtain this set of rings and avoiding repeated cases in the study, only are considered rings obtained by taking positive steps through jumps $\{1,2 k+$ $1\}$ or by taking positive steps through jumps $\{1,-(2 k+1)\}$. Therefore, for the rest of the paper, a $(2 k+1)$-ring embedded on a $C_{N}(1,2 k+1)$ is a cycle such that, if $\left\{n_{1}, n_{2}, \ldots, n_{2 k+1}\right\}$ is its set of nodes, then one of the following assertions must be true:

$-n_{i+1}=n_{i}+1 \bmod N$ or $n_{i+1}=n_{i}+(2 k+1) \bmod N, \forall i \in\{1,2, \ldots, 2 k+1\}$.

$-n_{i+1}=n_{i}+1 \bmod N$ or $n_{i+1}=n_{i}-(2 k+1) \bmod N, \forall i \in\{1,2, \ldots, 2 k+1\}$.

To simplify the notation we represent a $(2 k+1)$-ring as a vector whose coordinates are in the set $\{0,1\}$ or in $\{0,-1\}$, depending on the type of $(2 k+1)$ ring. If we are considering a $(2 k+1)$-ring in a $C_{N}(1,2 k+1), 0$ represents a (positive) step in jump 1, 1 represents a step in $2 k+1$ and -1 a step in $-(2 k+1)$. For example, the 7 -tuple $(1,0,0,1,0,0,1)$ denotes the 7 -ring $\{0,7,8,9,16,17,18\}$ in $C_{25}(1,7)$.

Using this notation above, we consider the following sets of $(2 k+1)$-rings:

$-A_{1}^{k}=\left\{\overline{\lambda_{1}}, \overline{\lambda_{2}}, \ldots, \overline{\lambda_{k}}\right\}$ where $\bar{\lambda}_{i}=(\overbrace{0, \ldots, 0}^{i}, \overbrace{1, \ldots, 1}^{k}, \overbrace{0, \ldots, 0}^{k+1-i})$, for $i=$ $1,2, \ldots, k$

$-A_{2}^{k}=\left\{\overline{\mu_{1}}, \overline{\mu_{2}}, \ldots, \overline{\mu_{k}}\right\}$ where $\bar{\mu}_{i}=(\overbrace{-1, \ldots,-1}^{i}, \overbrace{0, \ldots, 0}^{k}, \overbrace{-1, \ldots,-1}^{k+1-i})$, for $i=1,2, \ldots, k$.

Then, for every node $n$ of $C_{N}(1,2 k+1)$ there exists an element in $A_{1}^{k} \cup A_{2}^{k}$ containing $n$. This set of $2 k$ rings gives us a complete picture of the graph from node 0 . An example of this rings from node 0 in $C_{25}(1,7)$ can be seen in Fig. 4. 


\begin{tabular}{|c|c|c|c|c|c|c|c|c|}
\hline 1 & 8 & 15 & 22 & 4 & 11 & 18 & 0 & 7 \\
\hline 0 & 7 & 14 & 21 & 3 & 10 & 17 & 24 & 6 \\
\hline 24 & 6 & 13 & 20 & 5 & 9 & 16 & 23 & 5 \\
\hline 23 & 5 & 12 & 19 & 19 & 8 & 15 & 22 & 4 \\
\hline 22 & 4 & 11 & 18 & 0 & \multicolumn{1}{|c|}{} & 14 & 21 & 3 \\
\hline 21 & 3 & 10 & 17 & 24 & 6 & 13 & 20 & 2 \\
\hline 20 & 2 & 9 & 16 & 23 & 5 & 12 & 19 & 1 \\
\hline 19 & 1 & 8 & 15 & 22 & 4 & 11 & 18 & 0 \\
\hline 18 & 0 & 7 & 14 & 21 & 3 & 10 & 17 & 24 \\
\hline
\end{tabular}

Fig. 4. Characteristic Set of Rings for Node 0 on a $C_{25}(1,7)$ Graph.

Now, we consider the union of subsets of $(2 k+1)$-rings for all the nodes in the graph. It can be shown that this union has $2 N$ different elements (each ring from node 0 is repeated exactly $k$ times). Hence, the graph has been decomposed into a set of $2 N(2 k+1)$-rings, or dense rings, providing a complete picture of the graph.

In a recent paper of the authors [12], it was also proved that, for any integer $k$, there is a non-dense $C_{2 k^{2}}(1,2 k-1)$ graph, which can be seen as a collection of $2 N=4 k^{2} 2 k$-rings, or non-dense rings. The value of the integer $k$ corresponds to the diameter of both graphs and totally defines both topologies.

Finally, we can conclude that some specific members of the family of optimal circulant graphs of degree four are a particular evolution of the traditional ring topology or circulants of degree two.

\section{References}

1. A. Adám. "Research problem 2-10". J. Combinatorial Theory, 393, pp. 1109-1124, 1991.

2. NR. Adiga et al. "An Overview of the BlueGene/L Supercomputer", Supercomputing 2002, November 2002.

3. B.W. Arden and H. Lee. "Analysis of Chordal Ring Networks". IEEE Transactions on Computers, Vol. C-30, No. 4, pp. 291-295, 1981.

4. R. Beivide, E. Herrada, J.L. Balczar and A. Arruabarrena. "Optimal Distance Networks of Low Degree for Parallel Computers". IEEE Transactions on Computers, Vol. C-40, No. 10, pp. 1109-1124, 1991. 
5. J.-C. Bermond, F. Comellas and D.F. Hsu. "Distributed Loop Computer Networks: A Survey". Journal of Parallel and Distributed Computing, Vol. 24, pp. 2-10, 1995.

6. B. Bose, B. Broeg, Y. Known and Y. Ashir. "Lee Distance and Topological Properties of k-ary n-cubes". IEEE Transactions on Computers, Vol. 44, No. 8, pp. 1021-1030, 1995.

7. W.J. Bouknight, S.A. Denenberg, D.E. McIntyre, J.M. Randall, A.H. Sameh and D.L. Slotnick. "The Illiac IV System". Proc. IEEE, Vol. 60, No. 4, pp. 369-388. 1972.

8. M.A. Fiol, J.L. Yebra, I. Alegre and M. Valero. "A Discrete Optimization Problem in Local Networks and Data Alignment". IEEE Transactions on Computers, Vol. 36, No. 6, pp. 702-713, 1987.

9. K. Huber. "Codes over Tori". IEEE Trans. on Information Theory, Vol. 43, No. 2, pp. 740-744. March 1997.

10. L.E. LaForge, K.F. Korver and M.S. Fadali. "What Designers of Bus and Network Architectures Should Know about Hypercubes", IEEE Transactions on Computers, Vol. 52, No. 4, pp. 525-544, April 2003.

11. C. M. Lau and G. Chen. "Optimal Layouts of Midimew Networks". IEEE Transactions on Parallel and Distributed Systems, Vol. 7, No. 9, pp. 954-961, 1996.

12. C. Martínez, R. Beivide, C. Izu and J. Gutierrez. "Distance-Hereditary Embeddings of Circulant Graphs". Proc. of IEEE Int. Conf. on Information Technology: Coding and Computing (ITCC-2003). pp. 320-324. Las Vegas, 2003.

13. S. Mukherjee, P. Bannon, S. Lang, A. Spink and D. Webb. "The Alpha 21364 Network Architecture". IEEE Micro, Vol. 22, No. 1, pp. 26-35, 2002.

14. V. Puente, C. Izu, J.A. Gregorio, R. Beivide, J.M. Prellezo and F. Vallejo. "Rearranging Links to Improve the Performance of Parallel Computers: The Case of Midimew Networks". Proc. of International Conference on Supercomputing. ICS'2000. pp. 44-53. 2000.

15. R. S. Wilkov. "Analysis and Design of Reliable Computer Networks". IEEE Trans. On Communications, Vol. 20, 660-678.1972.

16. C.K. Wong and D. Coppersmith. "A Combinatorial Problem Related to Multimodule Memory Organizations". Journal of the ACM, Vol. 21, No. 3, pp. 392-402. 1974.

17. Y. Yang, A. Funashashi, A. Jouraku, H Nishi, H Amano and T. Sueyoshi, "Recursive Diagonal Torus: An Interconnection Network for Massively Parallel Computers". IEEE Transactions on Parallel and Distributed Systems, Vol. 12, No. 7, pp. 701-715, 2001. 